\title{
Prevalence of Syphilis among People living with HIV (PLHIV) attending a tertiary care hospital in coastal south India
}

\author{
Deepak Madi', Basavaprabhu Achappa', Poonam Vernakar², Unnikrishnan Bhaskaran ${ }^{3}$, \\ John T Ramapuram ${ }^{3}$, Soundarya Mahalingam ${ }^{1}$ \\ ${ }^{1}$ Associate Professor, ${ }^{2}$ Intern, ${ }^{3}$ Professor, Kasturba Medical College, Mangalore (Manipal University)
}

Background: Syphilis and HIV are both transmitted sexually so people can be infected with both diseases. Co-infection of syphilis and HIV alters the course of both the diseases. Venereal disease research laboratory (VDRL) test is a non-treponemal test, used commonly in Antiretroviral Therapy Centres (ART) in India to detect syphilis. The aim of our study was to determine the prevalence of syphilis in HIV-infected patients attending a tertiary care hospital using VDRL test. Materials and Methods: Using a cross-sectional study design 113 HIV positive patients were included. VDRLtest was used to screen for syphilis. The collected data was analyzed using SPSS version 11.5. Results: Among the 113 patients screened $69(61 \%)$ were males and $44(39 \%)$ were females.Majority of the patients had acquired the disease through heterosexual transmission (92.9\%). VDRL test was negative in all 113 patients. Conclusion: The seroprevalence of syphilis in coastal South India using VDRL is very less.Specific tests like Fluorescent Treponemal Antibody Absorption test (FTAABS) or Treponema pallidum hemagglutination Assay (TPHA) would be beneficial to detect asymptomatic syphilis in ART centers.

Key Words: Syphilis, HIV/AIDS, VDRL
Access this article online

Website:

http://nepjol.info/index.php/AJMS DOI: 10.3126/ajms.v6i6.11623

\section{INTRODUCTION}

The incidence of syphilis is rising all over the world, partly due to the increased transmission in HIV patients. ${ }^{1}$ Syphilis and HIV are both transmitted sexually so people can be infected with both diseases. HIV has several effects on the clinical presentation and diagnosis of syphilis. Syphilis increases the risk of HIV acquisition by causing genital ulcers $^{2}$ and it increases HIV transmission among HIV patients by increasing the amount of viral shedding.

Serological tests are used commonly to detect syphilis. Venereal disease research laboratory (VDRL) test is a non treponemal test, used commonly in Antiretroviral Therapy Centres in India (ART) for screening of syphilis due to its simplicity and low cost. There is hardly any data from India documenting the prevalence of syphilis using VDRL test. The aim of this study was to evaluate the prevalence of seroreactivity to syphilis among HIV-infected patients using VDRL test.

\section{MATERIALS AND METHODS}

A facility based cross-sectional study was carried out among 113 People Living with HIV (PLHIV) after obtaining ethics committee approval from the Institutional Ethics committee of Kasturba medical college Mangalore. The sample size was calculated based on an expected proportion of patients having HIV syphilis co-infection as $8 \%$, based on previous studies ${ }^{3}$ and absolute precision of $5 \%$ and confidence interval of $95 \%$. The study subjects were adult patients aged more than 18 years. HIV testing and interpretation was done according to NACO guidelines. 
During the study period of two months, 113 consecutive HIV patients who gave written informed consent participated in the study. The study was carried out at Kasturba Medical College hospital (tertiary health care centre) in Mangalore (South India). Patients who agreed to participate were interviewed by the investigators for 15 to 20 minutes. The interview was guided by a semi-structured questionnaire. VDRL test was used to detect syphilis.

Data collected was analyzed using SPSS Version 11.5 statistical software.Descriptive statistics were used to present the data.

\section{RESULTS}

A total of 113 HIV positive patients were screened for syphilis with VDRL as the tool. Among the 113 patients screened $69(61 \%)$ were males and $44(39 \%)$ were females (Table 1). Maximum number of patients belonged to the age group of 21-49 years i.e. 64 patients (Table 1). Majority of the study population was literate $(76 \%)$. Majority of patients $92.9 \%$ had acquired the disease heterosexually (Table 2) and $31.9 \%$ patients had CD 4 count more than 450 (Table 3). VDRL was negative in all 113 patients (Table 4).

\section{DISCUSSION}

The present study revealed that the prevalence of syphilis in coastal Karnataka is very less. Of the 113 patients studied VDRL test was negative in all the patients.

Serological investigations to diagnose syphilis are divided into non-treponemal tests and specific treponemal tests. VDRL and the rapid plasma reagin (RPR) are nontreponemal tests.VDRL is commonly used in Antiretroviral Therapy Centres (ART) to detect syphilis in India.All HIV positive patients should have baseline VDRL screening and if VDRL is reactive, the diagnosis should be confirmed by the specific treponemal test. ${ }^{4}$ Sensitivity of nontreponemal and treponemal tests for syphilis is approximately $75 \%$ in the primary stage and $100 \%$ in the secondary stage. ${ }^{5}$ False-positive and false-negative results on serologic tests for syphilis may be seen in HIV-infected persons., 5,6 Prozone reactions may affect VDRL. Prozone reactions result in false-negative reactions that occur due to high concentrations of target antibodies in the specimen. The incidence of the prozone phenomenon is high in the HIVinfected persons. ${ }^{3}$

In a study from India ${ }^{7}$ involving 88 HIV patients, eight patients $(9.1 \%)$ were reactive by VDRL and 42 patients were reactive by TPHA (47\%). All eight VDRL reactive patients were also reactive by TPHA.Turbadkar et al have

\begin{tabular}{|c|c|c|}
\hline \multirow{2}{*}{$\begin{array}{l}\text { Age } \\
\text { (years) }\end{array}$} & \multicolumn{2}{|c|}{ n (\%) } \\
\hline & Male & Female \\
\hline$<20$ & $1(.8)$ & $0(0)$ \\
\hline $21-49$ & $34(30)$ & $30(26.5)$ \\
\hline $50-69$ & $31(27.4)$ & $12(10.6)$ \\
\hline$>70$ & $3(2.6)$ & $2(1.7)$ \\
\hline Total & 69 & 44 \\
\hline
\end{tabular}

\begin{tabular}{lcc} 
Table 2: Mode of acquiring HIV & \\
\hline Mode of acquiring HIV & $\mathbf{n}$ & $\%$ \\
\hline Blood transfusion & 1 & 0.8 \\
Heterosexual & 105 & 92.5 \\
Vertical transmission & 1 & 0.8 \\
Unknown & 6 & 5.3 \\
\hline
\end{tabular}

\begin{tabular}{lcc}
\multicolumn{3}{l}{ Table 3: CD4 count of the study population } \\
\hline CD4 count (cells/ml) & N & $\%$ \\
\hline$<200$ & 23 & 20.4 \\
$200-249$ & 13 & 11.5 \\
$250-299$ & 7 & 6.2 \\
$300-349$ & 15 & 13.3 \\
$350-399$ & 6 & 5.3 \\
$400-449$ & 11 & 9.7 \\
$>450$ & 36 & 31.9 \\
Unknown & 2 & 1.8 \\
\hline
\end{tabular}

\begin{tabular}{lc}
\hline Table 4: VDRL results of study population \\
\hline VDRL & $\mathbf{N}$ \\
\hline Positive & 0 \\
Negative & 113 \\
\hline
\end{tabular}

recommended that in all cases of suspected syphilis two serological tests, i.e. one non-treporemal screening test, like VDRL and one specific treponemal test, like TPHA should be done for laboratory diagnosis of syphilis. ${ }^{\top}$ The prevalence of syphilis was $2.7 \%$ in a Brazilian study using VDRL test. ${ }^{8}$ In a study from Israel, $14 \%$ of HIV-infected patients had syphilis seroreactivity. ${ }^{9}$ The prevalence of HIV-syphilis co infection was $2.1 \%$ in Nigeria. ${ }^{10}$ According to Chow et al prevalence of HIV-syphilis co-infection was $2.7 \%$ among men who have sex with men (MSM). ${ }^{11}$ In a Spanish study of 1161 HIV-positive patients the baseline syphilis seroprevalence was $13 \% .{ }^{12}$ In contrast to these studies, we did not find any VDRL positive patients. Such observed difference could be due to different epidemiology of the infections in our study population.

Co-infection of syphilis and HIV alters the course of both the diseases. Syphilis causes transient increases in HIV viral load and decreases the CD4 count. ${ }^{13}$ These transient increases in viral load lead to the increased risk of HIV transmission. ${ }^{14}$ Detection and treatment 
of syphilis can, therefore reduce HIV transmission. ${ }^{15}$ In primary syphilis, HIV infected patients may present with more than one chancre. ${ }^{16}$ Co-infection with HIV increases the incidence of the neurological complications of syphilis. ${ }^{17}$

Our study had some limitations. Our study population did not include intravenous drug users and MSM. High rates of HIV/syphilis co-infection have been observed in MSM. So the prevalence of syphilis in our study may have been low. Repeat VDRL testing after 4-6 weeks was not done in our study to rule out the possibility of false negative results. We used a cross sectional study design and it has its own limitations.

\section{CONCLUSION}

The seroprevalence of syphilis in coastal South India using VDRL is very less. The serological diagnosis of syphilis may not be straight forward using non-specific treponemal tests. The efficacy of ART has resulted in increased survival of patients and their practice of unprotected sex especially in developing nations. As syphilis is still a prevalent infection in some developing countries, co-infection of HIV with syphilis should be considered. Specific tests like Fluorescent Treponemal Antibody Absorption test (FTA-ABS) or Treponema pallidum hemagglutination Assay (TPHA) would be beneficial to detect asymptomatic syphilis among HIV patients visiting ART centres even though they are costly.

\section{ACKNOWLEDGEMENT}

\section{ICMR STS}

\section{REFERENCES}

1. Ditzen AK, Braker K, Zoellner KH and Teichmann D. The syphilisHIV interdependency.Int J STD AIDS 2005; 16:642-643.
2. Arora PN and Sastry CV. HIV infection and genital ulcer disease. Indian J Sex Transm Dis 1992; 13: 71-73.

3. Kyriakis KP and Hadjivassiliou M. HIV-1 infection-associated risk factors among Sexually Transmitted disease patients in Athens, Greece: 1990 to 1996. Sex Transm Dis2000; 27(5):259-265.

4. Nayak $S$ and Acharjya B. VDRL test and its interpretation. Indian J Dermatol 2012; 57:3-8.

5. Rompalo AM, Cannon RO, Quinn TC and Hook EW. Association of biologic false-positive reactions for syphilis with human immunodeficiency virus infection. J Infect Dis 1992; 165: 1124-1126.

6. Haslett $P$ and Laverty $M$. The prozone phenomenon in syphilis associated with HIV infection. Arch Intern Med 1994; 154: 16431644.

7. Turbadkar D, Mathur M and Gaikwad S. Prevalence of syphilis among HIV seroreactive patients. Indian J Sex Transm Dis 2007; 28:91-93.

8. Signorini DJ, Monteiro MC, de Sá CA, Sion FS, LeitãoNeto HG, Lima DP, et al. Prevalence of HIV-syphilis coinfection in a university hospital in the city of Rio de Janeiro in 2005. Rev Soc Bras Med Trop 2007; 40:282-285.

9. Agmon-Levin N, Elbirt D, Asher I, Gradestein S, Werner B and Sthoeger Z. Syphilis and HIV co-infection in an Israeli HIV clinic: incidence and outcome. Int J STD AIDS 2010; 21:249-252.

10. Nnoruka EN and Ezeoke AC. Evaluation of syphilis in patients with HIV infection in Nigeria. Trop Med Int Health 2005; 10:58-64.

11. Chow EP, Wilson DP and Zhang L. HIV and Syphilis CoInfection Increasing among Men Who Have Sex with Men in China: A Systematic Review and Meta-Analysis. PLoS One 2011; 6:e22768.

12. Munoz-Perez MA, Rodriguez-Pichardo A and Camacho Martinez F. Sexually transmitted diseases in 1161HIV-positive patients: a 38-month prospective studyin southern Spain. J EurAcadDermatolVenereol1998; 11: 221-226.

13. Kofoed K, Gerstoft J, Mathiesen LR and Benfield T. Syphilis and human immunodeficiency virus (HIV)-1 co infection: influence on CD4 T-cell count, HIV-1 viral load, and treatment response. Sex Transm Dis 2006; 33:143-148.

14. Baeten JM and Overbaugh J. Measuring the infectiousness of persons with HIV-1: opportunities for preventing sexual HIV-1 transmission. Curr HIV Res 2003; 1:69-86.

15. Lynn WA and Lightman S. Syphilis and HIV: a dangerous combination. Lancet Infect Dis 2004; 4: 456-466.

16. Rompalo AM, Lawlor J, Seaman P, Quinn TC, Zenilman JM, Hook EW. Modification of syphilitic genital ulcer manifestations by coexistent HIV infection. Sex Transm Dis 2001; 28:448-454.

17. Zetola NM and Klausner JD. Syphilis and HIV Infection: An Update.Clin Infect Dis 2007; 44:1222-1228.

\footnotetext{
Authors Contribution:

DM, JTR and BA were the medical staffs who were involved in patient care. DM and UB provided scientific expertise for the article design. PV did the data collection. DM, JTR, BA, PV, UB and SM elaborated on the article and and revised the manuscript. All authors contributed to refinement of the article and approved the final manuscript.

Source of Support: Nil, Conflict of Interest: None declared.
} 